\title{
Frequency comb-based multidimensional coherent spectroscopy
}

\author{
Steven T. Cundiff ${ }^{1, *}$, and Bachana Lomsadze ${ }^{1}$ \\ ${ }^{1}$ Department of Physics, University of Michigan, Ann Arbor, 48109, Michigan, USA
}

\begin{abstract}
We present multidimensional coherent spectroscopy that utilizes frequency combs and multi-heterodyne detection. We demonstrate its capability to measure collective hyperfine resonances in atomic vapor induced by long-range dipole-dipole interactions.
\end{abstract}

Since its development about 2 decades ago, optical multidimensional coherent spectroscopy (MDCS) [1] has proven to be a powerful method to investigate structure, dynamics and many-body interactions. Optical MDCS uses a sequence of ultrafast laser pulses $(\mathrm{A}, \mathrm{B}, \mathrm{C})$ incident on the sample and records a non-linear (typically four-wave-mixing (FWM)) signal emitted by the sample as a function of the time delay(s) between the incident pulses. A multidimensional spectrum is constructed by calculating the Fourier transforms of the emitted signal with respect to the emission time and the delays between the pulses. Depending on the time ordering of the excitation pulses, a multidimensional spectrum can probe many-body interactions and provide important spectroscopic information. For instance, if the complex phase conjugated pulse interacts first with the sample $\left(A^{*}, B, C\right)$ then the corresponding multidimensional spectrum (referred to as a single-quantum spectrum) simultaneously provides homogenous and inhomogeneous linewidths of the samples resonances. Such a spectrum also shows coupling between resonances as offdiagonal peaks. If the complex phase conjugated pulse interacts last with the sample (B,C, $\mathrm{A}^{*}$ ) the multidimensional spectrum (referred to as a double-quantum spectrum) gives insight into many-body interactions [2]. However, until now the method, due to resolution and acquisition speed limitations, has only been used for samples with broad spectral features, particularly for semiconductor materials and molecules in solutions. The method has not been able to provide detailed information about weak many-body interactions (such as long-range dipole-dipole interactions) in atomic vapors. Recently we introduced a novel approach of multidimensional coherent spectroscopy that uses two frequency combs and a multi-heterodyne detection technique [3]. This combination allowed us to demonstrate rapid and the highest spectral resolution single-quantum multidimensional coherent spectroscopy $[4,5]$. Here we extend its applications for double-quantum multidimensional coherent spectroscopy and investigate dipole-dipole interactions in Rubidium atomic vapor $[6]$.

The experimental setup for frequency comb-based MDCS is schematically shown in Fig.1 (a). Two combs with different offset frequencies, produced from a single source comb (signal comb) using an Acousto-Optical Modulator, interact with a vapor of $\mathrm{Rb}$ atoms

* Corresponding author: cundiff@umich.edu 
$\left(100^{\circ} \mathrm{C}\right)$ in a collinear geometry and generate a FWM signal at different relative time delay. The emitted FWM signal is then spectrally separated in the RF domain after interfering it with a local oscillator (LO) comb that has a slightly different repetition rate [7]. A twodimensional spectrum is then generated by calculating the Fourier transforms of the signal with respect to the time delay between the excitation pulses and the time period during which it is emitted.

Figure 1 (b) shows the energy level diagrams of the D1 hyperfine lines of $87 \mathrm{Rb}$ and $85 \mathrm{Rb}$ that were probed in this experiment. Figure 1 (c) shows corresponding doublequantum two-dimensional spectrum. The spectrum was acquired with cross-linearly polarized excitation pulses and with the pulse ordering where the complex conjugated pulse interacts last with the sample. The diagonal peaks (along $(0,0) \mathrm{GHz}$ and $(10,20) \mathrm{GHz}$ line) correspond to couplings between the same hyperfine energy levels of two different atoms (inner white dashed box $85 \mathrm{Rb}$ and outer white dashed box $87 \mathrm{Rb}$ ) whereas the off-diagonal peaks show the coupling between different hyperfine energy levels of the same and different isotopes. The elongation of the peaks along the diagonal suggest that the FWM signal is predominately from the coupled atoms that have near zero velocity.

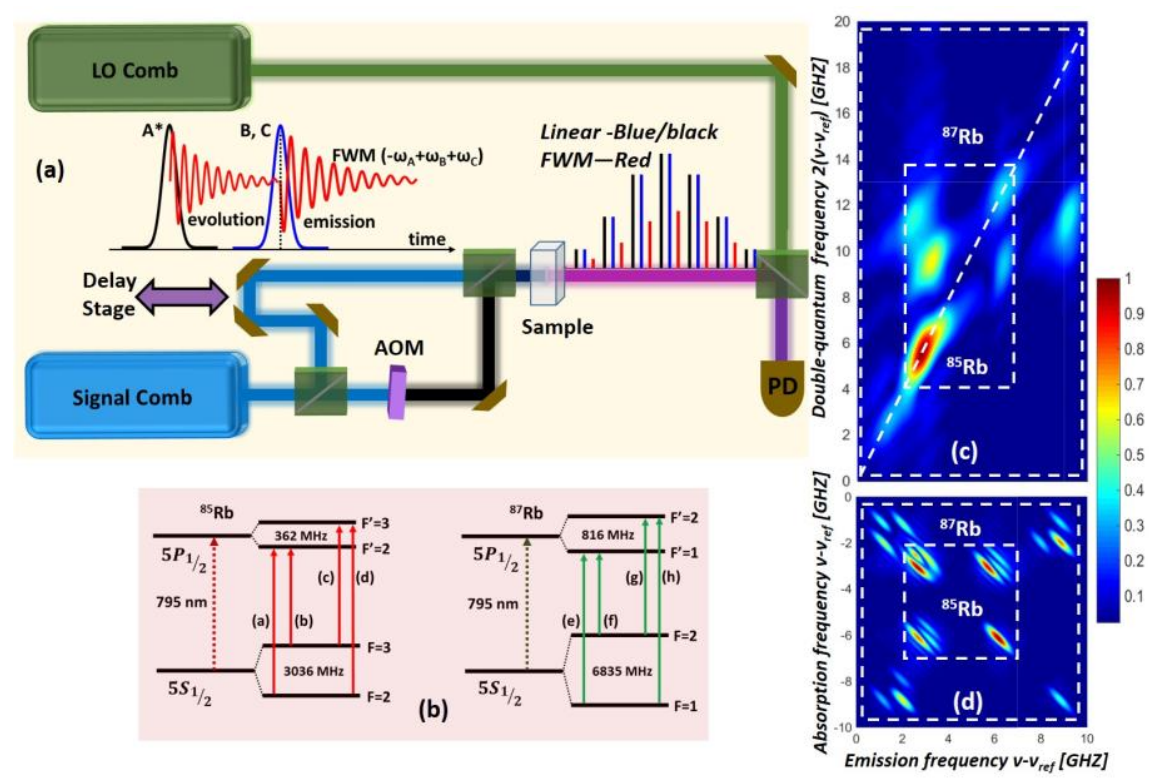

Fig 1. (a) Experimental setup. (b) energy level diagram of Rb D1 lines. (c) double-quantum two-dimensional spectrum. (d) single-quantum two-dimensional spectrum. $v_{\text {ref }}=377.103 \mathrm{THz}$, AOM Acousto-Optical Modulator, PD -photodetector.

We would like to emphasize that many-body interactions are accessible only using double-quantum coherent spectroscopy because it allows the measurement of the FWM signal that is only due to the interactions. These interactions are, in most cases, not accessible with other methods, including single-quantum MDCS. To demonstrate this point, we compared double-quantum $2 \mathrm{D}$ spectra to single-quantum $2 \mathrm{D}$ spectra shown in Fig. 2 (d) (taken by cross-linearly polarized excitation pulses). The spectra were taken with the pulse ordering that leads to formation of a photon echo (the complex conjugated pulse arrives first), which can be experimentally obtained by swapping the time order of the excitation pulses such that the AOM frequency shifted pulse (A) interacts first with the 
sample (Fig. 1 (a)). The diagonal elements (along $(0,0) \mathrm{GHz}$ and $(10,-10) \mathrm{GHz}$ line) correspond to FWM signals with the same absorption and emission hyperfine frequencies (a-h) for $87 \mathrm{Rb}$ (outer white dashed box) and $85 \mathrm{Rb}$ (inner white dashed box). They are diagonally elongated due to Doppler broadening. The cross-peaks, on the other hand, show all possible couplings between the hyperfine states within the same atom. In the photon echo excitation sequence the FWM signal due to the couplings of 2 different atoms via the dipole-dipole interaction is non-zero. However due to its weak strength compared to the FWM signal from individual atoms, the coupling peaks are not visible on $2 \mathrm{D}$ spectra. This shows that the single-quantum MDCS is not sensitive enough to probe the weak manybody interactions and measuring double-quantum spectra is required. Combination of single-quantum and double-quantum spectra makes frequency-comb based MDCS extremely powerful tool for obtaining a full spectroscopic information about the sample.

\section{References:}

[1] S. T. Cundiff, and S. Mukamel, "Optical multidimensional coherent spectroscopy", Physics today 66, 44-49 (2013).

[2] L. Yang, L. and S. Mukamel, "Two-dimensional correlation spectroscopy of twoexciton resonances in semiconductor quantum wells," Phys. Rev. Lett. 100, 057402 (2008). [3] I. Coddington, N. Newbury, and W. Swann, "Dual Comb Spectroscopy," Optica 3, 414$426(2016)$.

[4] B. Lomsadze, and S.T. Cundiff. "Frequency combs enable rapid and high resolution multidimensional coherent sepctroscopy," Science 357, 1389-1391 (2017).

[5] B. Lomsadze, and S.T. Cundiff. "Multi-heterodyne two dimensional coherent spectroscopy using frequency combs", Scientific Reports 7, 14018 (2017).

[6] B. Lomsadze and S.T. Cundiff, "Frequency-Comb Based Double-Quantum TwoDimensional Spectrum Identifies Collective Hyperfine Resonances in Atomic Vapor Induced by Dipole-Dipole Interactions,” Phys. Rev. Lett. 120, 233401 (2018).

[7] B. Lomsadze, and S.T. Cundiff. "Frequency comb-based four-wave-mixing spectroscopy," Opt. Lett. 42, 2346-2349 (2017). 